DOI: https://doi.org/10.24297/jap.v18i.8803

\title{
Far-Field, Radiation Resistance and temperature of Hertzian Dipole Antenna in Lossless Medium with Momentum and Energy Flow in the Far- Zone
}

\author{
Osama M. Abo-Seida ${ }^{a}$, N.T.M.Eldabe ${ }^{b}$, A.Refaie Ali*c, G. A. Shalaby ${ }^{c}$ \\ ${ }^{a}$ Department of Mathematics, Faculty of Science, Kafr El-Sheikh University, Egypt. \\ ${ }^{b}$ Department of Mathematics, Faculty of Education, Ain Shams University, Cairo, Egypt. \\ ${ }^{c}$ Department of Mathematics and Computer Science, Faculty of Science, Menoufia University, Egypt. \\ ahmed.r.master@gmail.com*, ahmed.refaie@science.menofia.edu.eg
}

\begin{abstract}
:
A Far-field with calculation of intrinsic impedance, ohmic heating and antenna temperature of radiated ideal (Hertzian) dipole antenna have been discussed in free space and lossless background. Actually, there is great important to analysis the radiation resistance mechanism of a Hertzian dipole antenna in an infinite isotropic lossless medium. We also discussed the momentum and energy flow in electromagnetic fields with investigation that wavefront/phase velocity is equals to light speed in far zone. The temperature of lossless Hertzian antenna is equal to brightness temperature $T_{o}$.
\end{abstract}

keyword: Hertzian dipole; Antenna temperature; Ohmic heating; Radiation resistance; Lossless media; Far field

\section{Introduction}

The radiation resistance output from a Hertzian dipole antenna plays a vital rule in modeling the radiated power into surrounding lossless medium on account of non dissipation of energy. In case of the surrounding environment a round the Hertzian antenna is infinite; then, the radiation resistance take non-zero value which is tantamount to power absorption. So, one can perhaps believe that in lossless background, all the radiated power is delivered to infinity. Because of choosing the lossless media; then, we can measure the apparent dissipation due to infinity radiated power propagation by the radiation resistance of the Hertzian dipole antenna.

Some sensors feel with temperature raising due to waves that fall on them. One of the thermal issues 12 is to care of temperature gradients in the manufacture of antennas. The loss of power associated with radiation resistance results in emission of electromagnetic radiation is due to ohmic heating of the antenna.

There are an literature review on Hertzian radiation in different media. Tai and Collin [2] have studied the radiation in a dissipative medium due to Hertzian dipole. Radiation resistance of antennas has been examined by Tsao [3] in lossy media. Mirmoosa et al [4] have explained the Physical meaning of radiation resistance of the Hertzian dipole antenna in lossy and lossless backgrounds. Because of the importance of applications of energy and momentum flow of Hertzian dipole antenna; Marcano and Diaz [1] calculate the total instantaneous energy velocity with harmonic excitation of Hertzian antenna in the far and near zones as function of radial distance. Flow of energy as well as flow of momentum has been studied by Mcdonald [5] in the near zone of a Hertzian dipole.

The research seeks to analysis the Hertzian dipole antenna and its radiation with a new perspective aims to calculating the radiation resistance, Poynting's vector, intrinsic impedance, far field and Ohmic heating of the antenna of Hertzian dipole. Also, In our paper, we care about the flowing in far -zone for the energy and the momentum. We also checked 
that velocity of wavefront / phase in far zone is equal to light speed. Finally, we obtain a form for antenna temperature from the brightness temperature.

\section{Discussions about the Hertzian dipole antenna}

Magnetic potential at point $Q$ given as a vector by :

$$
\overrightarrow{A_{h}}=\frac{\mu d \ell[I]}{4 \pi r} \hat{a_{z}}
$$

where, $\hat{a_{z}}$ take the same current direction and $d \ell \ll \frac{1}{10} \lambda$ (wave length). The current time to reach to $Q$ is $T=\frac{r}{v}=r \sqrt{\mu \epsilon}$, where, $v$ is propagation speed. when we neglect $\exp (j \omega t)$ then, $[I]$ become:

$$
[I]=R e\left\{I_{o} e^{-j \beta r}\right\}
$$

and by using the spherical coordinates, the components of $\vec{A}$ are: $A_{r h}=A_{z h} \cos \theta, A_{\theta h}=-A_{z h} \sin \theta, A_{\phi h}=0$ where $A_{z h}$ take the formula:

$$
A_{z h}=\frac{I_{o} \mu e^{-j \beta r}}{4 \pi r} d \ell
$$

Now, Magnetic flux density $\overrightarrow{B_{h}}=\mu \vec{H}_{h}=\nabla \times \overrightarrow{A_{h}}$. Most influential component of magnetic flux density is $H_{\phi h}$ become :

$$
H_{\phi h}=I_{o} \sin \theta e^{-j \beta r} d \ell 4 \pi\left[\frac{j \beta}{r}+\frac{1}{r^{2}}\right]
$$

we note in the bracket that the first term depend on $1 / r$ so, it called radiation field or far field. But the other term depend on $1 / r^{2}$ so, it be induction field or near field. If $r \beta \gg 1 \Rightarrow r \gg \frac{\lambda}{2 \pi}$ and if assumed that $r$ is very large then $1 / r^{2}$ can be neglected and the Far field will be created so on,

$$
H_{\phi h}=I_{o} \sin \theta e^{-j \beta r} j \beta d \ell 4 \pi r
$$

The electric component can be given in lossless dielectric medium by Maxwell equation as:

$$
\nabla \times \vec{H}_{h}=(\sigma+j \omega \varepsilon) \overrightarrow{E_{h}}=j \omega \epsilon_{o} \epsilon_{r} \overrightarrow{E_{h}}
$$

where, $\sigma=0 ; \varepsilon=\epsilon_{o} \epsilon_{r} ; \mu=\mu_{o} \mu_{r}$. From eqs.(5) and(6):

$$
j \omega \epsilon_{o} \epsilon_{r} \overrightarrow{E_{h}}=I_{o} e^{-j \beta r} . j \beta d \ell \sin 2 \theta 4 \pi r^{2} \sin \theta \hat{a_{r}}-I_{o} e^{-j \beta r}\left(\beta^{2}\right) d \ell \sin \theta 4 \pi r \hat{a_{\theta}}
$$

because of $E_{r h}$ is inversely proportional to $r^{2}$ then, it is a component of near field but, $E_{\theta h}$ is inversely proportional to $r$ then, it is far field component. $E_{\theta h}$ become:

$$
E_{\theta h}=\frac{\beta}{\omega \epsilon_{o} \epsilon_{r}}\left[I_{o} d \ell e^{-j \beta r} .(j \beta) \sin \theta 4 \pi r\right]=\frac{\beta}{\omega \epsilon_{o} \epsilon_{r}} H_{\phi h}
$$

Intrinsic impedance of Lossless dielectric medium $\eta$ calculated as :

$$
\eta=\frac{E_{\theta h}}{H_{\phi h}}=\frac{\beta}{\omega \epsilon_{o} \epsilon_{r}}
$$

Poynting vector:

$$
\vec{P}_{o}=R e\left\{E_{\theta h} \times H_{\phi h}\right\}=\eta \beta^{2} I_{o}^{2} d \ell^{2} \sin ^{2} \theta 32 \pi^{2} r^{2} \hat{a_{r}}
$$

where, $\hat{a_{r}}$ remains in the direction of propagation of electromagnetic wave. 


\section{Radiation Calculations}

Now, we represent the radiations in $E / H$. In particular radiations, electrical scalar potential take the form:

$$
V(t)=\frac{1}{4 \pi} \int_{v} \rho_{v}\left(t-\frac{r}{v}\right) r \epsilon_{o} \epsilon_{r} d v, \quad \text { volts }
$$

Again, the magnetic vector potential as function of $t$ is:

$$
\overrightarrow{A(t)}=\frac{\mu_{o} \mu_{r}}{4 \pi} \int_{v} \vec{J}\left(t-\frac{r}{v}\right) r d v
$$

And by using this formula for antenna such that for any point in space $P(\rho, \theta, \phi)$; consider that the current becomes in z-direction where, $I d z \hat{a_{z}}$ is current element of $\overrightarrow{A(t)}$ and its direction in the same direction of $\vec{I}$ and $\vec{J}$; hence,

$$
A_{z}=\frac{\mu I \cos \left(\omega\left(t-\frac{r}{v}\right)\right)}{4 \pi r} d z
$$

refers to the retarded vector magnetic potential. The components of magnetic field intensity are: $H_{r}=0, H_{\theta}=0$ and $H_{\phi}$ remains has non- zero value so, $\nabla \times \vec{H}$ have components only in $r$ and $\theta$ directions and these components have been derived from the following equation:

$$
\begin{gathered}
\vec{E}=\frac{1}{\epsilon_{o} \epsilon_{r}} \int(\nabla \times \vec{H}) d v \\
E_{r}=\frac{I \cos \theta}{2 \pi \epsilon_{o} \epsilon_{r}}\left[\frac{\cos \omega\left(t-\frac{r}{v}\right)}{r^{2} v}+\frac{\sin \omega\left(t-\frac{r}{v}\right)}{r^{3} \omega}\right] d z \\
E_{\theta}=\frac{I \sin \theta}{4 \pi \varepsilon}\left[\frac{-\omega \sin \omega\left(t-\frac{r}{v}\right)}{r v^{2}}+\frac{\cos \omega\left(t-\frac{r}{v}\right)}{r^{2} v}+\frac{\sin \omega\left(t-\frac{r}{v}\right)}{\omega r^{3}}\right] d z
\end{gathered}
$$

we notice from above that: $H_{\phi}$ is directly proportional to $\frac{1}{r}$ and $\frac{1}{r^{2}}$ while, $E_{r}$ depends on $\frac{1}{r^{2}}$ and $\frac{1}{r^{3}}$, and $E_{\theta}$ counts on $\frac{1}{r}, \frac{1}{r^{2}}$ and $\frac{1}{r^{3}} \cdot r$ is the radial distance of antenna; so, the radiation depended on $r$ so in case of area which is very much closer from current element ( antenna) i.e. $r<1$ so, $\frac{1}{r}$ refers to radiation but, $\frac{1}{r^{2}}$ and $\frac{1}{r^{3}}$ refers to induction.

\section{Radiation Resistance and power radiated}

By Balanis; density of average power is written as:

$$
\overrightarrow{P_{\rho, a v g}}=\frac{\eta \ell \sin \theta}{8 r^{2}}\left[1-\frac{j}{(r k)^{2}}\right]\left|\frac{I_{o}}{\lambda}\right|^{2}
$$

Power of radiation

$$
P_{\text {rad }}=\int \overrightarrow{\rho, a v g} \cdot \overrightarrow{d s}=40\left(\frac{d \ell}{\lambda}\right)\left(\pi I_{0}\right)^{2}
$$

As $I=I_{o} \cos \omega t, I_{r m s}=I_{o} / \sqrt{2}$; then,

$$
P_{\text {rad }}=I_{r m s}^{2} R_{\text {rad }}
$$

where, $R_{\text {rad }}$ is the radiation resistance for hertizian dipole antenna in free space or in applied lossless conditions.

\section{ohmic heating of the Hertzian dipole antenna}

If the resistance of a resistor is $R$; then, the average of ohmic heating power is:

$$
P_{\text {heat }}=<I_{o}^{2} R \cos ^{2} \omega t>=\frac{1}{2} R I_{o}^{2}
$$




\section{Application: Radiation into Lossless background}

Let us consider that there is a Hertzian dipole antenna with current amplitude $I_{o}$ and length $L$ immersed in an infinite isotropic lossless medium. we assume the medium is not magnetically; hence, $\mu=\mu_{o}$. In lossless media, the conductivity $\sigma=0$, which is effective factor. while, the relative permittivity as a complex value is:

$$
\varepsilon=\epsilon_{o}-j \epsilon_{o o}=\epsilon_{o}-\frac{\sigma j}{\epsilon_{o} \omega}=\epsilon_{o}
$$

Also, the refractive index $n$ is a complex value calculated by relation $n=\sqrt{\varepsilon}=n_{o}-j n_{o o}=n_{o}$ which is a positive real number. There is an important basis for construction of lossless medium which is: $\epsilon_{o o}=\frac{\sigma}{\epsilon_{o} \omega}=2 n_{o} n_{o o}=0$; because $n_{o o}$ tends to 0 for lossless media. As we mentioned in eq.(19) that the power transported from idealized dipole is $P_{\text {rad }}=I_{r m s}^{2} R_{\text {rad }}=n_{o}\left(\frac{40 d \ell}{\lambda}\right)\left(\pi I_{o}\right)^{2}$ where $R_{\text {rad }}$ is the radiation resistance in case of lossless surrounding medium. This medium quite not absorbs the power, therefore $P_{a b s}$ often approaches to zero for lossless background as $\sigma$ vanish for it. Also, the following relation vanishes:

$$
P_{a b s}=\frac{\sigma}{2} \int_{v}|E|^{2} d v=0
$$

Another way to calculate the power delivered from the source by the equation:

$$
P_{\text {rad }}=\frac{1}{2} \oint(\vec{E} \times \vec{H}) \cdot d s=\frac{1}{2}\left(I_{o} \pi\right)^{2}\left(\frac{80 d \ell}{\lambda}\right) n_{o}
$$

and this agree with the previous calculations of the power.

\section{Energy flow in far zone of a Hertzian dipole antenna}

Now, we determine the energy flow and it's density in electromagnetic fields of an idealized hertizian (point) oscillating electric dipole in far zone i.e. $r \gg \lambda$; where, the radiation field patterns are clearly appeared in that case. Both of electric field $E_{f}$ and magnetic field $H_{f}$ produced from ideal Hertzian electric dipole; whose moment is $m \cos \omega t$ in system of spherical coordinates $(r, \theta, \phi)$ are given in the following equations (24) and (25). With considering that z-axis take the dipole $m$ direction and the angle between $r$ and $m$ is $\theta$. so, the radiation fields are bigger that the other components of $H$ and $E$ as following:

$$
\begin{aligned}
& \overrightarrow{E_{f}}=-m k^{2} \sin \theta \cos (k r-\omega t) r \hat{\theta} \\
& \vec{H}_{f}=-m k^{2} \cos (k r-\omega t) \sin \theta r \hat{\phi}
\end{aligned}
$$

\section{Density of Energy}

Energy density which stored in vacuum or electromagnetic field is"

$$
U_{d}=\frac{1}{8 \pi}\left(E_{f}^{2}+H_{f}^{2}\right)=m^{2} k^{4}(1+\cos 2(k r-\omega t))(1-\cos 2 \theta) 16 \pi r^{2}
$$

\section{Energy flow}

Because of there is an energy flow out from the antenna. then, the radiated power be created and we can use Poynting's vector $P_{o}$ as an usual electrodynamic measure to estimate the a mount of energy flow into the surrounding medium and determined by the equation:

$$
P_{o}=\frac{c}{4 \pi}\left(\vec{E}_{f} \times \vec{H}_{f}\right)=<P_{o}>+c K^{4} m^{2} \cos 2(k r-\omega t) \sin ^{2} \theta 8 \pi r^{2} \hat{r}
$$


the second term of the above equation can be vary between positive and negative; so, part of energy can be flow inwards like outwards. The first term is time -averaged Poynting's vector and it equal to:

$$
<P_{o}>=c m^{2} k^{4} \sin ^{2} \theta 8 \pi r^{2} \hat{r}
$$

where, we have computed that: $\left\langle\cos ^{2}(k r-\omega t)>=\frac{1}{2}\right.$, while $<\cos 2(k r-\omega t)>=<\sin 2(k r-\omega t)>=0$. Poynting's vector is connected with the angular distribution of the radiated power in time-average by:

$$
\frac{<d P_{o}>}{d}=<P_{o}>\hat{r}=\frac{m^{2} k^{4} c \sin ^{2} \theta}{4 \pi}
$$

we now achieve continuity equation by Poynting vector in time-averaged scale that corresponds to flow of energy as following:

From eq.(26) we get:

$$
\nabla \cdot P_{o}+\frac{\partial U_{d}}{\partial t}=0
$$

$$
\frac{\partial U_{d}}{\partial t}=\omega m^{2} k^{4} \sin ^{2} \theta \sin 2(k r-\omega t) 4 \pi r^{2}
$$

while, from eq.(27) and eq.(28) we get:

$$
\nabla \cdot P_{o}=\frac{\left(-r k \sin 2(k r-\omega t)-\cos ^{2}(k r-\omega t)\right)}{4 \pi r^{4}} c k^{4} m^{2} \sin ^{2} \theta
$$

Therefore, the equation of continuity for far fields has been satisfied. so,

$$
\nabla \cdot\left(P_{o}\right)_{f}+\frac{\partial\left(U_{d}\right)_{f}}{\partial t}=0
$$

\section{Momentum flow in far zone of a Hertzian dipole antenna}

Poynting's vector plays double role for estimation both of energy flow and flow of momentum density that saved in the electromagnetic field. Momentum density can be calculated in case of oscillating dipole by:

$$
M_{\varrho}=\frac{E \times H}{4 \pi c}=c^{-2} P_{o}=(1+\cos 2(k r-\omega t)) r^{2} k^{4} m^{2} \sin ^{2} \theta 8 \pi c
$$

\section{Flux of momentum}

Momentum flux tensor $\Pi_{m}=($ momentum density $) *($ its velocity) as:

$$
\Pi_{m}=c M_{\varrho}=k^{4} m^{2} 4 \pi r^{2}\left(1-\cos ^{2} \theta\right) \cos ^{2}(k r-\omega t)
$$

where, $c$ is radiation fields speed which it radially propagates. The change rate of momentum density with respect to time $\frac{\partial M_{\varrho}}{\partial t}$ has a force density dimensions. Maxwell illustrated the forces associated with electromagnetic fields in terms of the stress tensor $S$ as:

$$
S_{i j}=\frac{E_{i} E_{j}+H_{i} H_{j}}{4 \pi}-\delta_{i j} U_{d}
$$

the volume force density $f=\nabla \cdot S=\frac{\partial M_{\varrho}}{\partial t} \Rightarrow$

$$
\frac{\partial M_{\varrho}}{\partial t}-\nabla \cdot S=0
$$

$\Rightarrow$

$$
\Pi_{m}=-S=\delta_{i j} U_{d}-\frac{E_{i} E_{j}+H_{i} H_{j}}{4 \pi}
$$

The momentum flux density that results from radiation fields is :

$$
\left(\Pi_{m}\right)_{f}=m^{2} k^{4}(1-\cos 2 \theta)(1+\cos 2(k r-\omega t)) 16 \pi r^{2}
$$




\section{Wavefront/phase velocity}

we take the wavefront of magnetic field due to the fields are transverse magnetic. So, when the electric hertzian dipole oscillate, $H_{f}$ vanishes at spherical surfaces. Hence, this leads to:

$$
H_{f}=\frac{-k^{2} m \sin \theta}{r} \cos (k r-\omega t) \hat{\phi}=0
$$

$\Rightarrow \cos (k r-\omega t)=0 \Rightarrow t=\frac{k}{\omega} r-\frac{\pi}{2 \omega}$, where $k=\omega / c$ is wave number. Consider phase speed denoted by $v_{P}$ calculated by:

$$
v_{p h}=\frac{1}{d t / d r}=1 k / \omega=c
$$

this result agree with physical meaning; where in the far zone, the phase velocity is equal to light speed.

\section{Thermal Emission and Antenna Temperature}

A thermal noise from the surrounding environment is an important influence that affect on systems of wireless communications. Thermal noise may be an interference or a signal. When the electrons collide with different particles, result from this collision an electromagnetic radiation that causes a thermal noise. The thermal intensity of propagation of thermal radiation in line of transmission is:

$$
I_{t} \cong T K
$$

where, $T$ here is refers to a brightness temperature. Johnson noise designated a Gaussian voltage $V_{J . G}$ for Thevenin circuit of load $Z_{o}=R$ which it can be radiated $T k B$ within the bandwidth $B[\mathrm{~Hz}]$ nethermost a connected transmission line. $R$ refers here to existence of a resistor $\mathrm{R}$ in temperature degree $T$. Thermal noise power here is:

$$
P_{t . n .}=T K B
$$

Hence, The thermal voltage at root-mean-square $V_{t h, r m s}$ within bandwidth $B$ is Johnson noise as:

$$
V_{t h, r m s}=2 \sqrt{T K V R B}
$$

Any a Hertzian dipole antenna connected to a transmitting line receives a thermal noise power $T_{A} k B$ from the surrounding space. Here, $T_{A}$ is denoted as the antenna temperature where it is average of weight that gained from temperature of brightness $T_{B}$, So $T_{A}$ defined as:

$$
T_{A}=\int_{4 \pi} \frac{T_{B} F}{4 \pi} d=T_{o}
$$

In case of lossless antenna and the brightness temperature $T_{B}=T_{o}$ then, $F(\theta, \phi)=H(\theta, \phi) \Rightarrow T_{A}=T_{o}$ as, $\int_{4 \pi} H(\theta, \phi) d=4 \pi ;$. Typically, The thermal noise adds $\sim 132 \mathrm{~K}$ from the environment to temperature of the antenna.

\section{Discussion and conclusion}

We have studied the Hertzian dipole antenna and its radiation with a new perspective. Furthermore, we have calculated the radiation resistance, Poynting's vector, intrinsic impedance, far field and Ohmic heating of the antenna of Hertzian dipole. Also, In our paper, we have interested with the flowing in the far -zone for the momentum and the energy. Finally, we have checked that the velocity of wavefront /phase in far zone is equal to light speed. As a result of our discussion, we conclude with some of important points as: 
- Possibility of increasing thermal radiation whenever the radiation that resulted from dipole antenna increases.

- Divergence of the absorbed power occurred in case of lossless medium.

- The absorbed power in an infinite lossless space approached to zero only when $n_{\text {oo }}$ (imagine part of complex refractive index $n$ ) tends to zero. There is no dissipation or lose of power in the "vacuum" and the infinite radiation occurred throughout the whole space.

- Poynting's vector is perpendicular on electric and magnetic fields and depend on the distance $r$, angle $\theta$ and time averaged Poynting's vector.

- The input resistance in lossy medium is the same of radiation resistance of lossless background in case of radiated Hertzian dipole antenna.

- Continuity equationis satisfied in Case of $P_{o}$ and $U_{d}$; also, in case of $M_{\varrho}$ and $S$ in far zone.

- If the real part of refractive index $n_{o}=0$; then, there is no any radiation resistance.

- In case of lossless Hertzian antenna; It's temperature is equal to brightness temperature $T_{o}$.

- Wavefront/Phase velocity match with light speed in far zone.

- As an important observation that Johnson noise does not add in case of lossless transmitting line.

Funding Not applicable.

\section{Availability of data and materials}

Not applicable.

\section{Authors' contributions}

All authors jointly worked on the results and they read and approved the final manuscript.

\section{Competing interests}

The authors declare that they have no competing interests.

\section{References}

[1] Marcano, D., Diaz, M, .: Energy Transport Velocity for Hertzian Dipole. IEEE Anten. Propa.So.Int. Symposium. vol.10, 2017-2020(2006).

[2] Tai, C. T., Collin, R. E.,.: Radiation of a Hertzian dipole immersed in a dissipative medium. IEEE Trans. Antenn. Propag. vol 48, 1501-1506(2000)

[3] Tsao, C. K. H.,.: Radiation resistance of antennas in lossy media. IEEE Trans. Antenn. Propag. 19, 443-444(1971)

[4] Mirmoosa, M. S., et.al.,.: Physical meaning of the dipole radiation resistance in lossless and lossy media. .arXiv:1901.02777v1 [physics.class-ph]. (2019)

[5] McDonald, Kirk T.: Flow of energy and momentum in the near zone of a Hertzian dipole. NJ 08544,(2014) 
[6] Poynting, J.H.: On the transfer of energy in the electromagnetic field, Phil. Trans. Roy. Soc. London. 175, 343(1884)

[7] Schantz,H. G,.: Electromagnetic energy around Hertzian dipoles. In IEEE Antennas and Propagation Magazine. vol. 43, no. 2, 50-62 (2001)

[8] Michalski, Krzysztof.: On the Alternative Vector Potential Formulation of the Sommerfeld Half-Space Problem. IEEE Antennas and Wireless Propagation Letters. 1-1(2017)

[9] Yehia, H.M.,.:Regular precession of a rigid body (gyrostat) acted upon by an irreducible combination of three classical fields. Journal of the Egyptian Mathematical Society 25, Issue 2, 216-219(2017)

[10] Hayat, A., Faryad, M., On the radiation from a Hertzian dipole of a finite length in the uniaxial dielectric medium. OSA Continuum. 2, 1411(2019)

[11] Anyutin, N., Malay,I., Malyshev,A.: Calculation of phase center of arbitrary electromagnetic radiation sources in near field zone. IEEE East-West Design and Test Symposium (EWDTS). 1-4 (2019)

[12] Abo-Seida, Osama M., et.al.: Far-Field Radiated From a Vertical Magnetic Dipole in the Sea with a rough upper surface. IEEE Transactions on geoscience and remote sensing, 44(2006)

[13] Chapter 11.: Common Antennas and Applications. Electromagnetics and Applications. Spring (2009), MIT OpenCourseWarehttp://ocw.mit.edu

[14] Senthil, R., et al.: Effect of the Phase Change Material in a Solar Receiver on Thermal Performance of parabolic Dish Collector. Thermal Science. 21, 2803-2812(2017)

[15] C. G. Moschovitis, K. T. Karakatselos, E. G. Papkelis et al., "Scattering of electromagnetic waves from a rectangular plate using an enhanced stationary phase method approximation," IEEE Transactions on Antennas and Propagation, vol. 58, no. 1, pp. 233-238, 2010.

[16] I. Arun and M. Venkatapathi, "Analysis of numerical solutions to sommerfeld integral relation of the half-space radiator problem," Applied Numerical Mathematics, vol. 106, pp. 79-97, 2016.

[17] S. T. Bishay, O. M. Abo-Seida, and H. S. Shoeib, "Analytical formulas for dipole radiation buried inside planar stratified media," in Proceedings of the International Conference on Aerospace Sciences and Aviation Technology (ASAT-16), Cairo, Egypt, May 2015. 


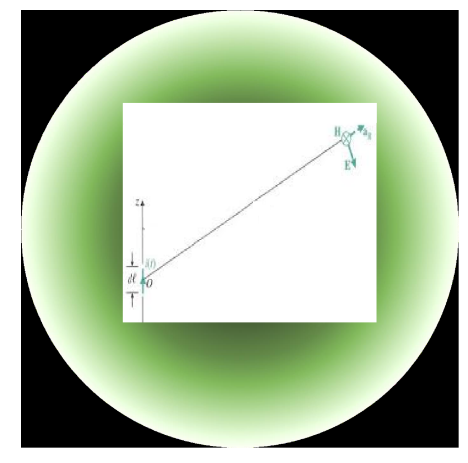

Figure 1: Hertzian dipole antenna immersed in Lossless medium 